\title{
The Study on the Anti-corrosion Performance of NiTi Alloy in Human Body Solution with the Fabricating Processes of Laser Irradiation and PDMS Modification
}

\author{
Chengjuan Yang ${ }^{1,2}$, Weiran Cao ${ }^{1,2}$, Zhen Yang ${ }^{1,2,3}$, Meng Wang ${ }^{1,2^{*}}$, Xiubing Jing ${ }^{1,2}$, Yanling Tian ${ }^{1,2,3}$ \\ 1. School of Mechanical Engineering, Tianjin University, Tianjin 300054, China \\ 2. Key Laboratory of Mechanism Theory and Equipment Design of Ministry of Education, Tianjin 300072, China \\ 3. School of Engineering, University of Warwick, Coventry CV4 7AL, UK
}

\begin{abstract}
This paper presents a new and safe method of fabricating super-hydrophobic surface on NiTi Shape Memory Alloy (SMA), which aims to further improve the corrosion resistance performance and biocompatibility of NiTi SMA. The super-hydrophobic surfaces with Water Contact Angle (WCA) of $155.4^{\circ} \pm 0.9^{\circ}$ and Water Sliding Angle (WSA) of $4.4^{\circ} \pm 1.1^{\circ}$ were obtained by the hybrid of laser irradiation and polydimethylsiloxane (PDMS) modification. The forming mechanism was systematically revealed via Scanning Electron Microscopy (SEM) and X-ray Photoelectron Spectroscopy (XPS). The anti-corrosion of samples was investigated in Simulated Body Fluid (SBF) via the potentiodynamic polarization (PDP) and Electrochemical Impedance Spectroscopy (EIS) tests. PDMS super-hydrophobic coatings showed superior anti-corrosion performance. The $\mathrm{Ni}$ ions release experiment was also conducted and the corresponding result demonstrated that the super-hydrophobic samples effectively inhibited the release of Ni ions both in electrolyte and SBF. Besides, biocompatibility was further analyzed, indicating that the prepared super-hydrophobic surfaces present a huge potential advantage in biocompatibility.
\end{abstract}

Keywords: wettability, laser texturing, corrosion resistance, mechanism, human body liquid, Ni ions release

Copyright $(\subset$ The author(s) 2021.

\section{Introduction}

Nitinol Shape Memory Alloy (NiTi SMA), possessing special performance such as wear resistance, super-elasticity, Shape Memory Effect (SME) and biocompatibility has drawn wide attention in recent years ${ }^{[1]}$. As a new potential material, NiTi SMA is increasingly employed in medical applications ${ }^{[2]}$, for instance medical equipment. However, as the near equiatomic material, the corrosion of NiTi SMA will inevitably appear due to the long-term usage, resulting in $\mathrm{Ni}$ ions release into the physiological environment ${ }^{[3]}$. It has been reported that $\mathrm{Ni}$ ions at a high level in the human body can induce the occurrence of adverse reactions such as toxicity, anaphylaxis, chronic inflammation and so on ${ }^{[4]}$, which severely restricts the development of NiTi SMA as medical equipment. Hence, enhancing the corrosion resistance and biocompatibility of NiTi SMA plays a vital role in its application in medicine and has attracted plenty of attention in recent years.
Various surface modification techniques have been proposed to prevent the material from corrosion. A protective coating is commonly applied on NiTi SMA for anti-corrosion, such as calcium phosphate-based coatings, carbon-based coatings, $\mathrm{TiN}$ or $\mathrm{TiO}_{2}$ coatings and various composite coatings ${ }^{[5,6]}$. Tohidi et al. created hydroxyapatite (HAp) coatings on NiTi via pulsed electrodeposition under magnetic field. The corrosion behavior and bioactivity of HAp coatings were evaluated through electrochemical potentiodynamic polarization tests in Ringer's solution and immersion tests in Simulated Body Fluid (SBF). They have demonstrated that the HAp coatings with superior anti-corrosion and bioactive could be obtained via optimizing process parameters in the electrodeposition ${ }^{[7]}$. Kurtoglu et al. treated NiTi SMA in flowing ammonia at $700{ }^{\circ} \mathrm{C}$ to fabricate a protective TiN coating on NiTi SMAs. The coating could obviously mitigate the Ni ion release from NiTi SMAs after immersion in artificial saliva ${ }^{[8]}$. However, the former method of deposition coatings is high cost and inapplicable

\footnotetext{
*Corresponding author: Meng Wang

E-mail: mw77@tju.edu.cn
} 
for mass production, and the latter method of surface modification can alter the internal performance of NiTi SMA at high temperature. Therefore, more alternative strategies showing cost-effective and simple are eagerly exploited to enhance the corrosion resistance and biocompatibility of NiTi SMA in human body liquid and promote the large-scale usage of it.

Inspired by bionics, fabricating super-hydrophobic surface on materials has been regarded as an admirable technology of excellent anti-corrosion performance ${ }^{[9,10]}$. Previous literatures have demonstrated that the real contact area between super-hydrophobic interface and corrosive medium is extremely small on account of plenty of air pockets trapping underneath the laser-induced micro-nano structures ${ }^{[11,12]}$. According to previous researches, structuring rough surfaces with micro-nano structures and lowering surface energy have been summarized as the two methods to successfully fabricate super-hydrophobic surfaces ${ }^{[13,14]}$. Varied methods to structure requisite micro-nano structures have been proposed, including electrodeposition, self-assembly, chemical etching, electrospinning, sol-gel, anodizing, and spin coating ${ }^{[15-20]}$. The aforesaid processing approaches show poor practicability with respect to environmental pollution or high cost. Conversely, laser surface patterning is identified as an efficient and convenient method of constructing micro-nano structures ${ }^{[21,22]}$. Additionally, the controllable surface morphology can be acquired by altering laser processing parameters. Compared with aforesaid processing rough surface approaches, the laser-induced micro-nano structures showed superior mechanical stability ${ }^{[23]}$. Significantly, super-hydrophobic surfaces can be fabricated purely based on laser ablation ${ }^{[24,25]}$. However, this kind of super-hydrophobic surfaces is limited in industrial mass production due to the dependency of surrounding environment and the uncertainty of natural aging time for different kinds of materials. Besides, it can be easily damaged under high temperature or interaction with corrosive liquid ${ }^{[26,27]}$. Coating a chemical layer with low surface energy on the laser-induced surfaces has been regarded as the commonly used method to improve the processing efficiency and the stability of super-hydrophobic surfaces ${ }^{[28]}$. The surfaces exhibit splendid low-affinity to water via coating a layer of low surface energy materials, including fatty acids (e.g., stearic acid, myristic acid, lauric acid, etc.), fluoroakylsilane (FAS), octadecylamine, and so on ${ }^{[29,30]}$. Lu et al. created microstructures with various scales on the $316 \mathrm{~L}$ stainless steel surfaces using different nanosecond laser (ns-laser) parameters. Based on the further modification of FAS the super-hydrophobic surfaces with a high Water Contact Angle (WCA) of $160^{\circ} \pm 5^{\circ}$ and a small Water Sliding Angle (WSA) of $3^{\circ} \pm 0.5^{\circ}$ were obtained. The electrochemical tests revealed that the corrosion resistance of the biomimetic super-hydrophobic surfaces was effectively enhanced. Additionally, the laser-textured super-hydrophobic surfaces showed excellent self-cleaning performance ${ }^{[31]}$. The typical super-hydrophobic surface with a high WCA of $158.2^{\circ}$ was successfully fabricated in the Liu's group via laser irradiation followed by chemical etched and stearic acid modification on AZ31 magnesium alloy surfaces. Besides, the super-hydrophobic surfaces possessed good anti-corrosion, outstanding chemical and thermal stability $^{[32]}$. For the medical device, the modification process may cause harm to human body due to the perniciousness of toxic chemical materials. Hence it is urgent to obtain a green and health method to fabricate super-hydrophobic surfaces to meet the demand of security in medicine. Notably, the chemical modification process of polydimethylsiloxane (PDMS) is regarded as a green and health surface modification method ${ }^{[33]}$.

In this paper, a new method of fabricating secure super-hydrophobic surface was presented by the hybrid of nanosecond laser (ns-laser) irradiation and PDMS modification. The transformation mechanism of surface wettability was systematically investigated via the analysis of surface morphology and chemical composition. The aim of this study was to explore the corrosion resistance and biocompatibility of NiTi SMA coated with PDMS super-hydrophobic coatings. The electrochemical experiments were carried out to estimate the corrosion resistance of as-prepared samples in SBF. Meanwhile, the concentration of released Ni ions from as-prepared samples was measured by Inductively Coupled Plasma-Mass Spectrometry (ICP-MS) after immersion tests. Moreover, the cell viability was analyzed by investigating the release amount of $\mathrm{Ni}$ ions from samples after sterile immersion. The overview 


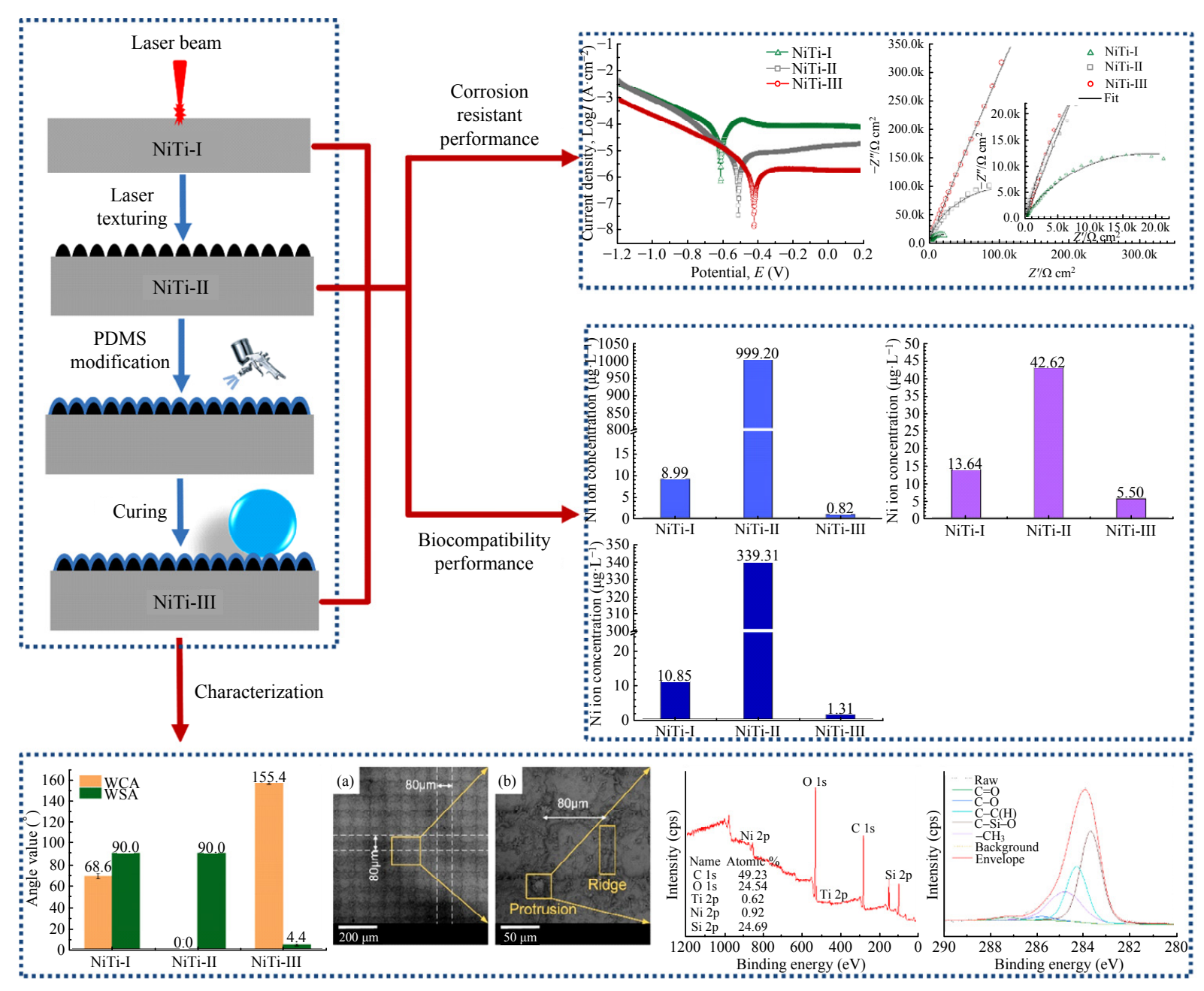

Fig. 1 The overview of this study.

of this study is shown in Fig. 1. The corresponding experimental results indicated that the super-hydrophobic coatings exhibited high performance of anti-corrosion, the inhibition of ions release and biocompatibility. This new approach for surface modification of NiTi is simple, efficient, cost-effective as well as low thermal effect, which can promote the promising prospect in industrial production.

\section{Experiment and method}

\subsection{Materials preparation}

The experiments were carried out on NiTi SMAs with a size of $10 \mathrm{~mm} \times 10 \mathrm{~mm} \times 0.8 \mathrm{~mm}$. NiTi SMAs were provided by Kejing Material Technology Co., Ltd, Hefei, China. To ensure the uniformity of surface, all the prepared samples were mechanically polished on a high-speed polishing machine until obtaining mirror surfaces. Then, all the polished samples were ultrasonically cleaned by acetone (Analytical Reagent, AR: > 99.5\%), ethanol (AR: 95\%) and deionized water for 15 min one after another in an ultrasonic cleaning tank to remove the impurity and oil stain on the surfaces. Finally, all the cleaned samples experienced vacuum drying process in a drying oven at $80{ }^{\circ} \mathrm{C}$ for $30 \mathrm{~min}$. The chemicals: The acetone (AR: $>99.5 \%$ ), ethanol (AR: 95\%), deionized water, SBF (SBF, Phygene) and Phosphate Buffer Solution (PBS) were provided by Jiangtian Chemical Technology Co. Ltd. The PDMS polymer cross-linking agent (Dow Corning from USA, Sylgard 184) was purchased from Alfa Aesar.

\subsection{Fabrication of micro-pillar arrays}

As shown in Fig. 2, the polished samples were irradiated by a ns-laser processing system (Han's Laser, 
China), which is consisted of an Ytterbium fiber laser source (IPG-YLP-20, Germany), laser beam expander, $\mathrm{XY}$-scan mirror and focusing lens. Nanosecond pulsed laser beam were generated by an Ytterbium fiber laser source and propagated along the path of light. The laser beam expander was utilized to decrease the divergence angle and expand beam diameter. The laser beam was focused onto the sample surfaces using the focusing lens with the focal length of $182 \mathrm{~mm}$. The processing parameters of the ns-laser were regulated and controlled by HL marking software: wavelength $(\lambda) 1064 \mathrm{~nm}$, average power $(P) 18 \mathrm{~W}$, pulse duration $(\tau) 50 \mathrm{~ns}$, repetition rate (f) $20 \mathrm{kHz}$, spot diameter $(\phi) 50 \mu \mathrm{m}$, and scanning speed (v) $500 \mathrm{~mm} \cdot \mathrm{s}^{-1}$. The samples were fixed on the working platform, and were ablated in two vertical directions, resulting in the ordered micro-pillar arrays. As in each direction, the laser beam moved in line-by-line scanning pattern. The distance of centers between two adjacent laser beams' scanning paths was set at $80 \mu \mathrm{m}$. The laser ablation process was performed at atmospheric environment with a temperature of $23^{\circ} \mathrm{C} \pm 3{ }^{\circ} \mathrm{C}$.

\subsection{Chemical modification with PDMS polymer}

Lots of residual debris was produced on fabricated surface during the laser ablation process. Hence, before chemical modification process, the samples were washed in ultrasonic bath containing deionized water for $5 \mathrm{~min}$, and then dried by compacted nitrogen gas. PDMS was mixed with the curing agent at room temperature, and then ethyl acetate was rapidly poured into polymer mixture and stirred continuously until the solution became transparent and homogeneous. The mass ratio of PDMS, curing agent and ethyl acetate in the modifier solution was 10:1:450. The obtained PDMS modifier was poured into the upper pot of the spray gun. Under the action of working pressure of $0.3 \mathrm{MPa}$, PDMS polymer was sprayed on the fresh laser-induced NiTi SMA surfaces with a height of $25 \mathrm{~cm}$. Then, samples were dried in an oven at $80{ }^{\circ} \mathrm{C}$ for half an hour. Finally, the typical super-hydrophobic surfaces were successfully processed on NiTi SMA samples. This fabrication process was shown in Fig. 3. The primary NiTi SMA samples without any processing treatment were abridged as NiTi-I. The laser-induced NiTi SMA samples were categorized into two groups according to whether the PDMS modifier solution was coated or not. NiTi-II: The samples were purely ablated by ns-laser but without

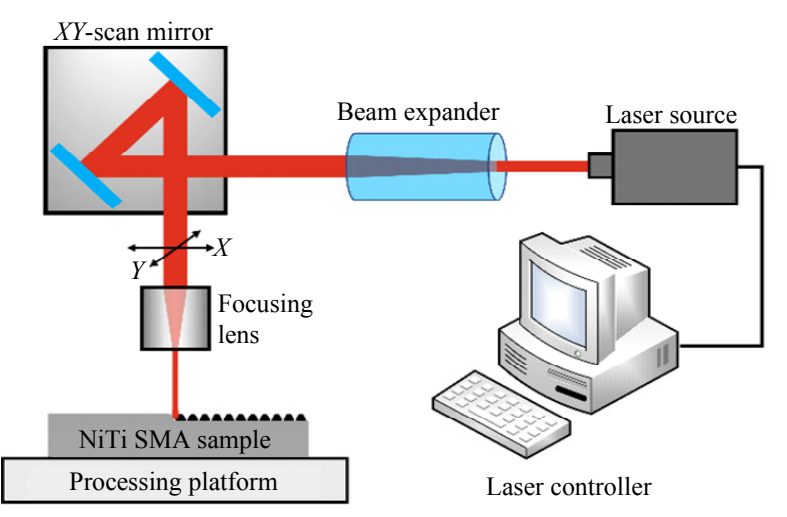

Fig. 2 Schematic diagram of integrated ns-laser system.

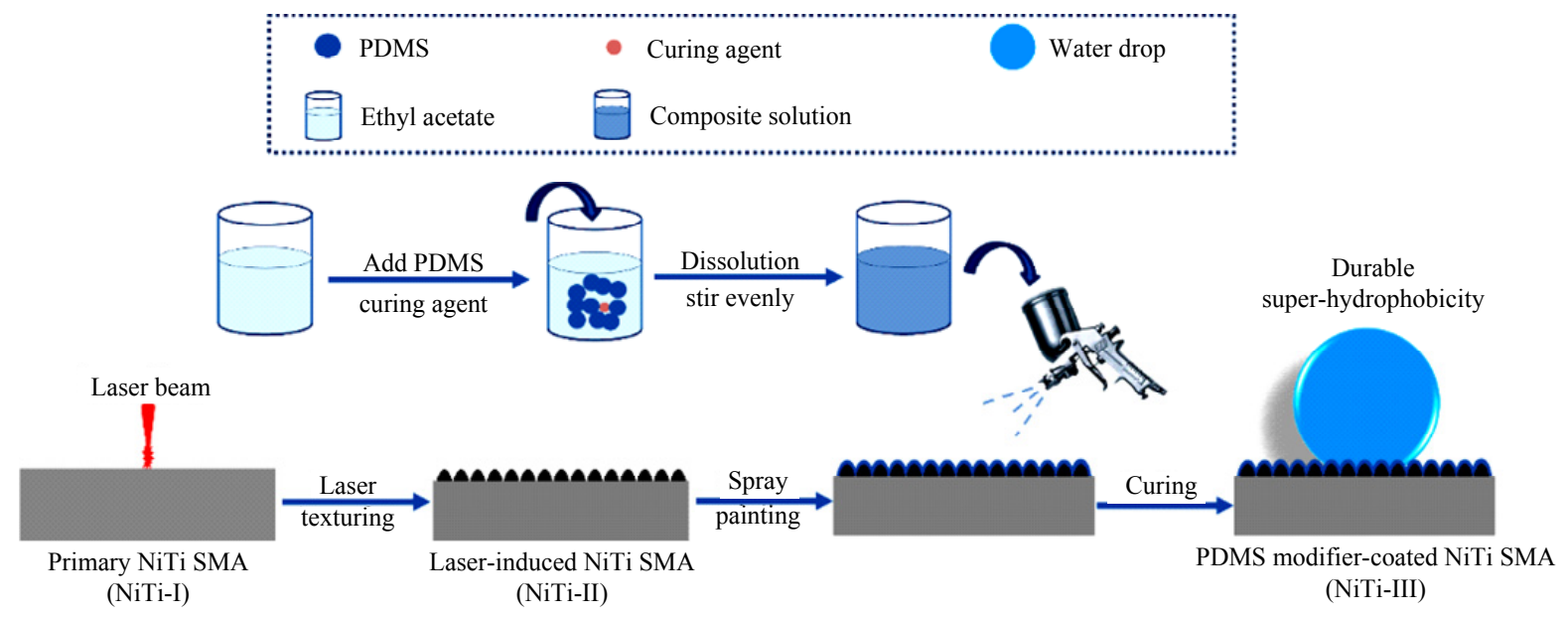

Fig. 3 Schematic diagram of durable super-hydrophobic surface fabrication process. 
Yang et al:: The Study on the Anti-corrosion Performance of NiTi Alloy in Human Body

Solution with the Fabricating Processes of Laser Irradiation and PDMS Modification

Table 1 Composition of modified SBF

\begin{tabular}{ccccccrrr}
\hline Component & $\mathrm{Na}^{+}$ & $\mathrm{K}^{+}$ & $\mathrm{Mg}^{2+}$ & $\mathrm{Ca}^{2+}$ & $\mathrm{Cl}^{-}$ & $\mathrm{HCO}_{3}{ }^{-}$ & $\mathrm{HPO}_{4}{ }^{2-}$ & $\mathrm{SO}_{4}{ }^{2-}$ \\
\hline Ionic concentrations $\left(\mathrm{mmol} \cdot \mathrm{L}^{-1}\right)$ & 142.0 & 5.0 & 1.5 & 2.5 & 103.0 & 10.0 & 1.0 & 0.5 \\
\hline
\end{tabular}

other treatment; NiTi-III: The samples were induced by ns-laser followed by PDMS modifier coating immediately.

\subsection{Surface characterization}

WCAs and WSAs were detected using a WCA goniometer (AST, VCA optima) to evaluate the surface wettability of investigated samples. Besides, surface micro-structures of the treated samples were observed through Scanning Electron Microscope (SEM: FEI, Quanta 250 FEG) and Leica microscope. 3D profiles and surface roughness were evaluated by a white confocal light microscope (CountourGT, Bruker). X-ray Photoelectron Spectroscopy (XPS: Thermo Fisher Scientific, Escalab 250Xi) was used to analyze the surface chemical compositions.

\subsection{Measurement and characterization}

The corrosion resistance of three kinds of samples was examined in SBF at room temperature and ambient air via electrochemical test. The composition of SBF is shown in Table 1. The electrochemical tests were conducted via an electrochemical workstation (CHI660E, China). Potentiodynamic polarization (PDP), Open Circuit Potential (OCP) and Electrochemical Impedance Spectroscopy (EIS) tests were performed in a typical three-electrode cell configuration: The treated samples, a Pt plate $(20 \mathrm{~mm} \times 20 \mathrm{~mm} \times 0.1 \mathrm{~mm})$ and a Saturated Calomel Electrode (SCE) were used as working electrode, counter electrode and reference electrode, respectively. The working electrode with $1 \times 1 \mathrm{~cm}^{2}$ surface area exposed to corrosion medium. The PDP tests were employed from $-1.2 \mathrm{~V}$ to $0.4 \mathrm{~V}$ at a scanning rate of $10 \mathrm{mV} \cdot \mathrm{s}^{-1}$. The samples were first immersed in SBF for $30 \mathrm{~min}$ before OCP tests to ensure OCP value steady. Then, the EIS tests were performed from $100 \mathrm{kHz}$ to $10 \mathrm{mHz}$ at the alternating current amplitude of $10 \mathrm{mV}$. ZSimPWin software was utilized to fit the EIS results. After electrochemical test, the inductively coupled plasma-mass spectrometry (ICP-MS: Agilent, 7800) was used to reveal the concentration of $\mathrm{Ni}$ ions in electrolyte.
In the $\mathrm{Ni}$ ions release test, the as-prepared samples were immersed in SBF for $4 \mathrm{~h}$ with the same conditions (the volume of medium, the number of samples, temperature and so on). In order to study the stability and biocompatibility of the samples under simulated vivo conditions, the sterile immersion tests were conducted in PBS for $2 \mathrm{~h}$ under with a temperature of $37^{\circ} \mathrm{C}$ and a humidified atmosphere of $5 \% \mathrm{CO}_{2}$ in an aseptic condition. Before the sterile immersion test, all samples were sterilized using $75 \%$ alcohol by volume and UV light in sequence. All measurements were conducted three times for results reproducibility and data reliability.

\section{Results and discussion}

\subsection{Surface wettability}

The static WCAs and WSAs of the fabricated samples were measured using sessile drop technique on the goniometer to evaluate surface wettability. Each measured value was averaged over three different locations. The results of Fig .4 showed that NiTi-I surfaces exhibited intrinsic hydrophilicity with a small WCA of $68.2^{\circ} \pm 2.3^{\circ}$ and high WSA of $90^{\circ}$. After ns-laser irradiation, NiTi-II surfaces showed super-hydrophilicity with the WCA below $10^{\circ}$. Satisfactorily, after chemical modification with PDMS on fresh laser-induced surfaces, the super-hydrophobic surfaces were successfully fabricated indicating that the super-hydrophobic surfaces can be obtained by the combined action of laser irradiation and chemical modification of PDMS polymer. The typical super-hydrophobic surfaces showed a high WCA of $155.4^{\circ} \pm 0.9^{\circ}$. Besides, an $8 \mu \mathrm{L}$ water droplet could immediately roll down from as-prepared super-hydrophobic surfaces with a low till angle of $4.4^{\circ} \pm$ $1.1^{\circ}$, which was regarded as the value of WSA. These results confirmed that the super-hydrophobic surfaces had low water adhesion.

Wenzel model was employed to reveal the transformation mechanism of surface wettability from theoretical perspective. The corresponding contact angle calculation equation of solid surface is shown in Eq. (1):

$$
\cos \theta_{\mathrm{r}}=R \cos \theta_{\mathrm{f}},
$$


where $\theta_{\mathrm{f}}$ is the intrinsic static WCA of a solid surface (that is, the WCA of the smooth surface); $\theta_{\mathrm{r}}$ denotes the apparent WCA on a rough solid surface; $R$ is the roughness factor, which is defined as the ratio between the actual surface area and projected area of a solid surface. Obviously, as far as a rough surface, $R$ is greater than 1 .

Eq. (1) implies that the ascent of surface roughness results in the enhancement of surface wettability. That is to say, a hydrophilic surface will be more hydrophilic and a hydrophobic surface will be more hydrophobic with the increase of surface roughness ${ }^{[34]}$. According to Table 2, the surface roughness signally increased after laser irradiation, resulting in the super-hydrophilicity of NiTi-II. The results coincided with the Wenzel model. Previous literature has demonstrated that the super-hydrophobic surface can be generally fabricated via the combined action of processing hierarchical structure and modifying low surface energy chemical materials ${ }^{[35]}$. In this study, the super-hydrophobic surfaces were successfully fabricated after coating a layer of PDMS polymer with low surface energy on laser irradiated surface. The corresponding mechanism was further investigated in sections $\mathbf{3 . 2}$ and $\mathbf{3 . 3}$.

\subsection{Surface morphology}

As shown in Fig. 5, surface morphology was obviously changed after ns-laser irradiation under the aforesaid processing parameters. The number of laser pulses per spot $(N)$ and the pulse overlap factor $\left(E_{\text {of }}\right)$ can be calculated via Eqs. (2) and (3):

$$
\begin{gathered}
N=\frac{\pi \phi}{4 l}, \\
E_{\text {of }}=\frac{2}{\pi \phi}\left[\phi \arccos \left(\frac{l}{\phi}\right)-l \sqrt{1-\left(\frac{l}{\phi}\right)^{2}}\right],
\end{gathered}
$$

where $\phi$ is spot diameter for laser beam; $1=v / f$ represents the pulse distance along laser scanning direction, $v$ $\left(\mathrm{mm} \cdot \mathrm{s}^{-1}\right)$ and $f(\mathrm{kHz})$ are scanning speed and repetition rate for ns-laser pulse, respectively. Putting the processing parameter into the above-mentioned formula, it can be calculated that $N$ was 2 and $E_{\text {of }}$ was $39.10 \%$.

Figs. $5 \mathrm{a}-5 \mathrm{~d}$ showed the SEM images of laser-induced NiTi SMA surfaces under different

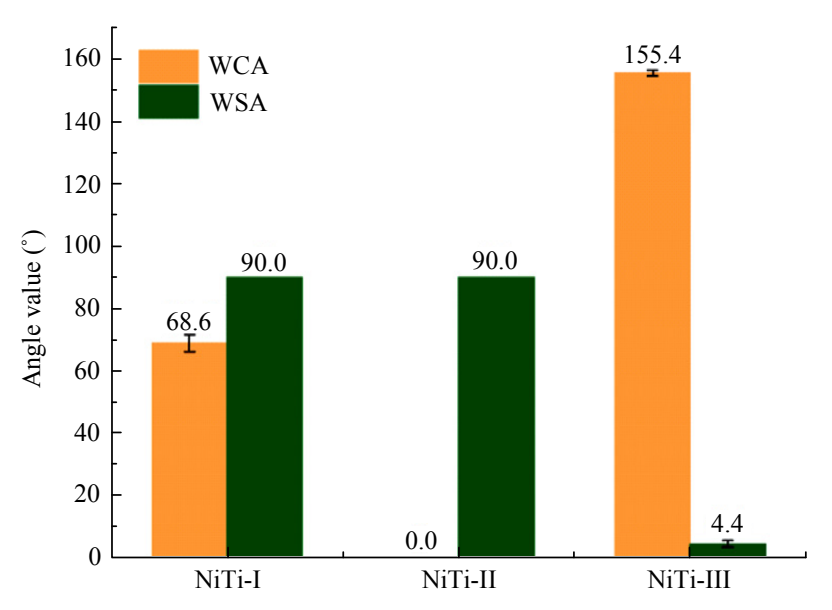

Fig. 4 WCAs and WSAs of NiTi-I, NiTi-II and NiTi-III samples surfaces.

Table 2 Surface roughness of NiTi-I, NiTi-II and NiTi-III samples

\begin{tabular}{cccc}
\hline Sample & NiTi-I & NiTi-II & NiTi-III \\
\hline Roughness $R_{\mathrm{a}}(\mu \mathrm{m})$ & 0.0524 & 8.7372 & 8.6753 \\
\hline
\end{tabular}

magnifications, respectively. According to Fig. 5a, the laser-induced NiTi SMA surface was filled with regular grid patterns or grooves. The generation of grooves was attributed to the line-by-line scanning of laser beam. The scanning cycle $(d)$ is defined as the distance between the two adjacent laser beam scanning paths, which is equal to the distance between the middle lines of two adjacent grooves in Fig. 5a. The enlarged image of a grid texture was shown in Fig. 5b. It can be clearly seen that grid textures consisted of basin-shaped pits, ridges and protrusions. Each protrusion was surrounded by grooves. As shown in Figs. 5c and 5d, the textured surface was covered in numerous droplets-shaped particles. The attendance of the particles was attributed to the high energy of laser processing resulting in the interfacial materials melting and splashing over the sample. The spraying materials with micro or nano size rolled and then re-solidified onto the sample surface. As a result, the particles irregularly deposited on the inside wall and the brim of grooves. The hierarchical rough structure facilitated the trap of large air pockets, which contributed to the suspension of water droplets on NiTi-III surfaces.

\subsection{Surface chemical composition}

In order to reveal the generation mechanisms of 

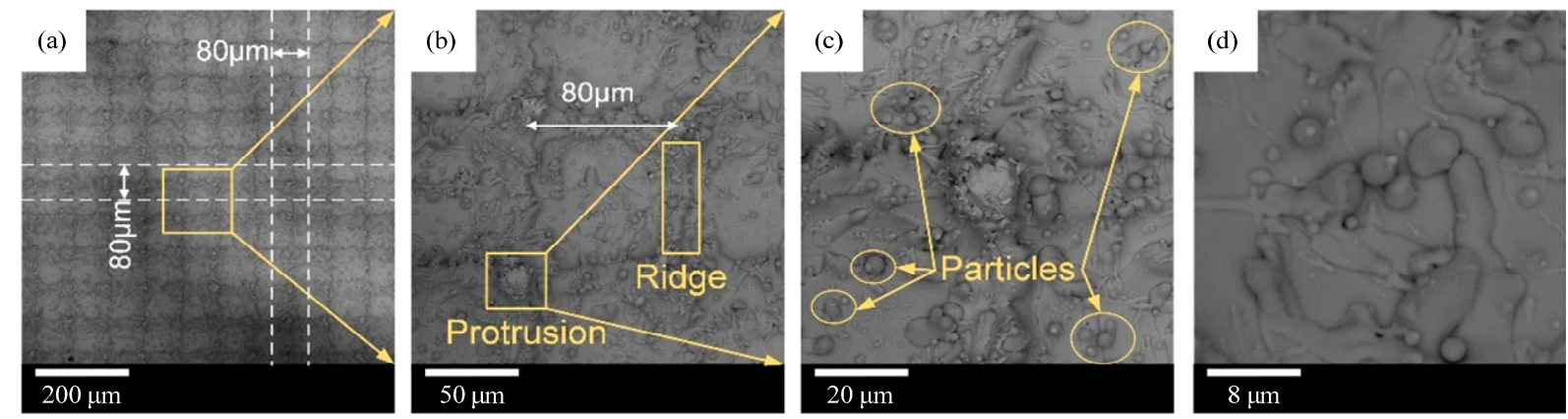

Fig. 5 SEM images of NiTi-III surfaces with different scales.

super-hydrophobic surfaces, XPS spectra were performed to analyze the surface chemical compositions of the fabricated samples. Fig. 6 exhibited the chemical composition of NiTi-I, NiTi-II and NiTi-III surfaces. According to the XPS spectra in Figs. 6a and 6b, NiTi-I and NiTi-II surfaces both contained four elements: $\mathrm{C}$ at $284.0 \mathrm{eV}, \mathrm{O}$ at $532.0 \mathrm{eV}$, Ti at $458.0 \mathrm{eV}$ and $\mathrm{Ni}$ at 855.0 $\mathrm{eV}$. The influence of other elements with very low content was ignored and only the spectra with binding energy between $100 \mathrm{eV}$ and $900 \mathrm{eV}$ in the XPS survey plots were considered. However, besides the above four elements, the additional element $\mathrm{Si}$ at $102.0 \mathrm{eV}$ obviously appeared on NiTi-III surfaces in Fig. 6c, indicating the attachment of silicon-based polymers material of PDMS polymer on laser processed rough surface. On the other hand, compared with NiTi-I, NiTi-III surfaces had lower content of $\mathrm{Ni}$ and $\mathrm{Ti}$. This phenomenon was attributed to the present of PDMS polymer film resulting in decreasing the relative amount of $\mathrm{Ni}$ and $\mathrm{Ti}$.

In order to have an insight of the formation mechanism of the super-hydrophobic film, the high resolution of $\mathrm{C} 1 \mathrm{~s}$ and $\mathrm{O} 1 \mathrm{~s}$ peaks were carried out based on the analysis of CasaXPS software. The high-resolution spectra of O 1s of NiTi-I and NiTi-III surfaces were presented in Figs. 6d and 6e, respectively. As shown in Fig. 6 d, the $\mathrm{O} 1 \mathrm{~s}$ spectrum of the NiTi-I surfaces contained three main functional groups. The peaks located at $531.2 \mathrm{eV}, 532.6 \mathrm{eV}$ and $529.8 \mathrm{eV}$, were regarded as the functional group $\mathrm{C}=\mathrm{O}, \mathrm{C}-\mathrm{O}$ and oxides (nickel oxide and titanium oxide), respectively. It could be found that nickel and titanium primarily existed in the forms of nickel and titanium oxides, respectively. According to the $\mathrm{O}$ 1s spectrum of NiTi-III surfaces in Fig. 6f, the $\mathrm{O} 1 \mathrm{~s}$ peak contained three additional functional groups. The peaks were located at $531.8 \mathrm{eV}, 532.5 \mathrm{eV}$ and $533.7 \mathrm{eV}$ referred to $\mathrm{O}-\mathrm{Si}-\mathrm{C}, \mathrm{Si}-\mathrm{O}-\mathrm{Si}$ and $\mathrm{Si}-\mathrm{OH}$ moieties, respectively ${ }^{[36]}$. The functional groups $\mathrm{O}-\mathrm{Si}-\mathrm{C}$ and $\mathrm{Si}-\mathrm{O}-\mathrm{Si}$ came from the PDMS polymer chains, which further verified that the PDMS had been successfully coated on fresh laser-induced surfaces after chemical modification process.

Fig. $6 \mathrm{~g}$ displayed the decompositions of $\mathrm{C} 1 \mathrm{~s}$ spectrum of NiTi-I surfaces. Three strong peaks $\mathrm{C}=\mathrm{O}$, $\mathrm{C}-\mathrm{O}$ and $\mathrm{C}-\mathrm{C}(\mathrm{H})$ located at $287.5 \mathrm{eV}, 285.6 \mathrm{eV}$ and $284.5 \mathrm{eV}$ were measured. Obviously, the carbon content should be $0 \%$ on NiTi-I. However, NiTi-I surfaces showed a strong signal of $\mathrm{C} 1 \mathrm{~s}$. The carbon element of NiTi-I surfaces might derive from three main sources: The liquid residues of acetone and ethanol during cleaning processes, the absorption of organic matters in air and the adhesion of the volatile oil from the vacuum chamber of the XPS apparatus. In the same way, the presence of the carbon element on NiTi-II surfaces might be due to the same reasons. Besides, the decomposition of pre-existing absorbed organic matters was another main source. As shown in Fig. 6h, the C 1s spectrum of NiTi-III surfaces contained five functional groups: $\mathrm{C}=\mathrm{O}, \mathrm{C}-\mathrm{O}, \mathrm{C}-\mathrm{C}(\mathrm{H}), \mathrm{C}-\mathrm{Si}-\mathrm{O}$ and $-\mathrm{CH}_{3}$. The functional group $\mathrm{C}-\mathrm{Si}-\mathrm{O}$ at $283.5 \mathrm{eV}$ and $-\mathrm{CH}_{3}$ at $285.0 \mathrm{eV}$ came from PDMS polymer film ${ }^{[37,38]}$. In general, the function group of $-\mathrm{CH}_{3}$ possessing strong nonpolar lowered the surface energy extremely, resulting in the change of wettability from super-hydrophilicity to super-hydrophobicity after coating a layer of PDMS polymer on laser-induced surfaces. Therefore, it can be concluded that the combined action of low surface energy of PDMS and laser-induced rough structures contributed to the generation of super-hydrophobic surfaces. 

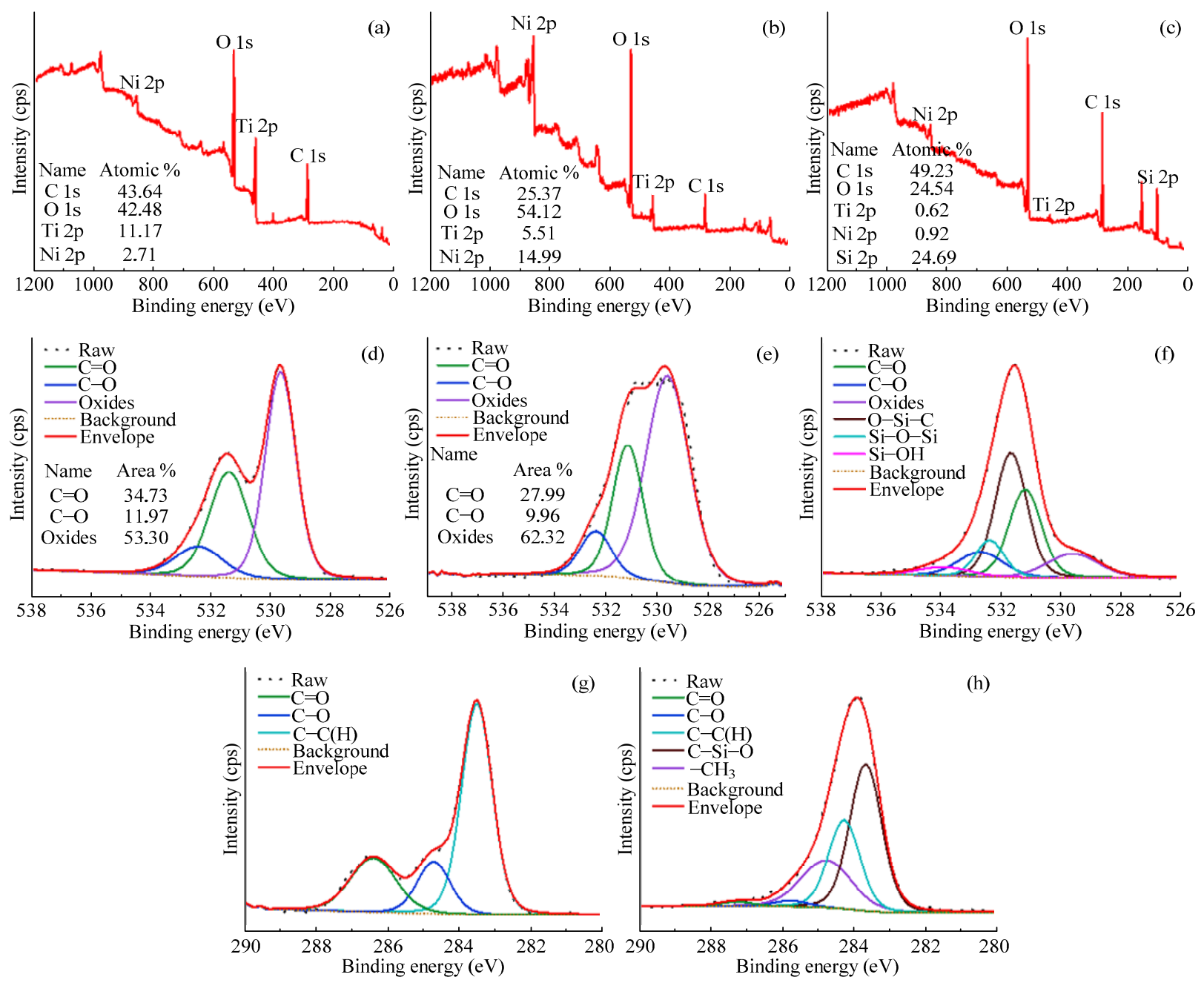

Fig. 6 XPS spectra of (a) NiTi-I, (b) NiTi-II, and (c) NiTi-II samples; the high-resolution O 1s spectra of (d) NiTi-I, (e) NiTi-II, and (f) NiTi-III samples; the high-resolution C 1s spectra of (g) NiTi-I, and (h) NiTi-III samples.

The generation of super-hydrophilicity after laser irradiation process might come from two main sources: (1) According to the XPS survey spectra in Fig. 6b, the oxygen content witnessed an apparent increase from $42.18 \%$ to $54.12 \%$ after the irradiation of ns-laser, regarding the oxidation reaction of titanium and nickel occurred during the laser ablation process. Besides, the generated titanium oxide and nickel oxide contained a great deal of atoms in the form of $\mathrm{Ti}^{4+}, \mathrm{Ni}^{2+}$ and $\mathrm{O}^{2-}$ after laser processing ${ }^{[26]}$. The undersaturated titanium, nickel and oxygen atoms serving as Lewis acid and base pairs resulted in the hydrophilicity of surface metallic oxides $^{[39]}$. (2) Obviously, the titanium and nickel atoms were electron-deficient. In order to gain a full octet electron, these atoms combined the hydrogen bonds from water molecules ${ }^{[40]}$ as shown in Fig. 7. As a result, the surface polarity significantly increased with extremely non-equilibrium, which further enhanced surface hydrophilicity.

\subsection{Electrochemical corrosion tests}

Previous literature has reported that super-hydrophobic surfaces exhibit superior corrosion resistance. Fabricating superhydrophobic coating on metals and alloys is known as an effective method to promote corrosion resistance. In this paper, a new method was proposed for the fabrication of secure super-hydrophobic surface by the hybrid of ns-laser irradiation and PDMS modification to enhance the corrosion resistance of NiTi SMA. The electrochemical test has attracted great attention to the investigation of corrosion resistance. PDP and EIS tests were employed to 


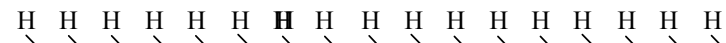

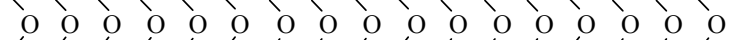

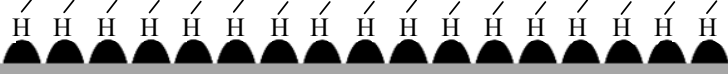

NiTi-II samples

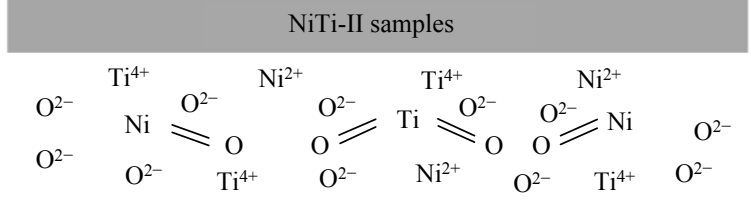

Fig. 7 Schematic illustration of hydroxylation process of NiTi-II surfaces.

explore and compare the corrosion resistance of NiTi-I, NiTi-II and NiTi-III samples in SBF, respectively.

\subsubsection{PDP curves}

The PDP curves of aforesaid samples were measured in SBF as shown in Fig. 8a. The corrosion current density $\left(I_{\text {corr }}, \mathrm{A} \cdot \mathrm{cm}^{-2}\right)$ and corrosion potential $\left(E_{\text {corr }}, \mathrm{V}\right)$ were calculated via extrapolating the linear portion of PDP curves to the intersection. The corrosion rate $(C R)$ was calculated according to formulas as given below ${ }^{[41]}$ :

$$
\begin{array}{r}
C R=\mathrm{K} \frac{I_{\text {corr }}}{\rho} E W, \\
E W=\frac{1}{\frac{n_{1} \cdot f_{1}}{W_{1}}+\frac{n_{2} \cdot f_{2}}{W_{2}}},
\end{array}
$$

where $\mathrm{K}$ is a constant, $\mathrm{K}=3.27 \times 10^{-3} \mathrm{~mm} \cdot \mathrm{g}$. $(\mu \mathrm{A} \cdot \mathrm{cm} \cdot \mathrm{year})^{-1}, \rho$ is the density of NiTi SMA in $\mathrm{g} \cdot \mathrm{cm}^{-3}$, $E W$ is the equivalent weight of NiTi SMA, $n, f$ and $W$ are the valence, mass fraction and atomic weight of the elements in NiTi SMA, respectively. Subscripts 1 and 2 represent Ni element and Ti element, respectively.

In the meanwhile, the corrosion inhibition efficiency $(\eta)$ of NiTi-I and NiTi-III samples was obtained using the following formula ${ }^{[20]}$ :

$$
\eta(\%)=\frac{I_{\text {corr }}^{\prime}-I_{\text {corr }}}{I_{\text {corr }}^{\prime}} \times 100 \%,
$$

where $I_{\text {corr }}$ and $I_{\text {corr }}$ represent the corrosion current density of the surfaces with and without the inhibition layer, respectively. The corresponding calculated results were summarized in Table 3. In the corrosion thermodynamics, the corrosion potential shows the difficult degrees of corrosion, the positive-going corrosion potential

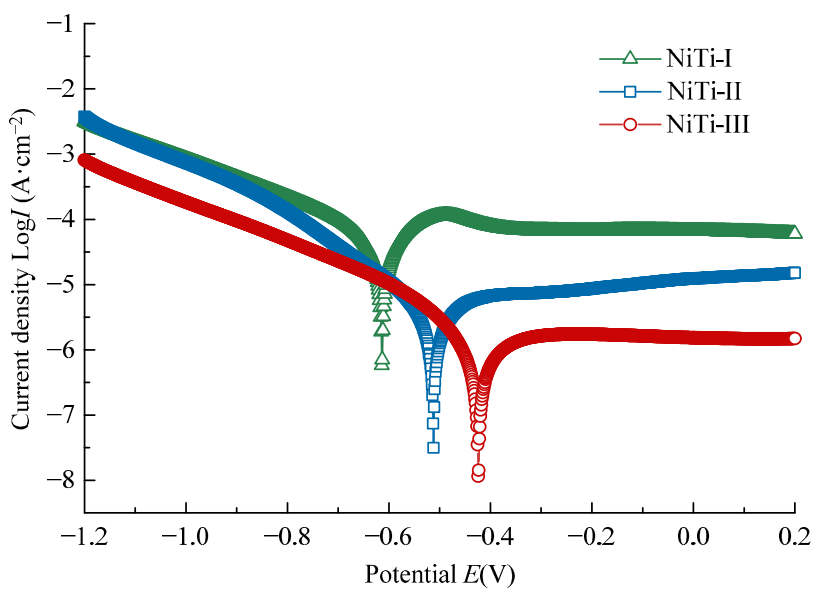

Fig. 8 PDP curves of NiTi-I, NiTi-II and NiTi-III samples in SBF.

Table 3 Corrosion current density $\left(I_{\text {corr }}\right)$, corrosion potential $\left(E_{\text {corr }}\right)$ and corrosion inhibition efficiency $(\eta)$ of NiTi-I, NiTi-II and NiTi-III samples

\begin{tabular}{ccccc}
\hline Sample & $I_{\text {corr }}\left(\mathrm{A} \cdot \mathrm{cm}^{-2}\right)$ & $E_{\text {corr }}(\mathrm{V})$ & $C R\left(\mathrm{~mm} \cdot \mathrm{year}^{-1}\right)$ & $\eta(\%)$ \\
\hline NiTi-I & $8.644 \times 10^{-5}$ & -0.614 & 0.74 & - \\
NiTi-II & $6.023 \times 10^{-6}$ & -0.512 & 0.052 & 93.0 \\
NiTi-III & $1.293 \times 10^{-6}$ & -0.424 & 0.011 & 98.5 \\
\hline
\end{tabular}

expresses the better corrosion resistance. The corrosion rate and the corrosion inhibition efficiency are determined from the corrosion current density of samples, that is to say, a lower corrosion current density is regarded as superior anti-corrosive performance. Therefore, the results indicated that NiTi-II surfaces presented better corrosion resistance behavior than NiTi-I surfaces. As expected, NiTi-III samples had the best anti-corrosive property among the three kinds of samples, demonstrating that fabricating super-hydrophobic surfaces through coating a layer of low surface energy of PDMS on laser-induced surfaces could obviously enhance corrosion resistance.

\subsubsection{EIS test}

As a complementary electrochemical experiment, EIS test was carried out in SBF to further investigate the corrosive resistance of as-prepared samples. The OCP versus time of NiTi-I, NiTi-II and NiTi-III samples in SBF was illustrated in Fig. 9a. According to the OCP curves, it can be apparent that NiTi-III showed the highest OCP values than that of other samples. This phenomenon indicated that NiTi-III might possess the largest anti-corrosion potential in SBF than other samples. 


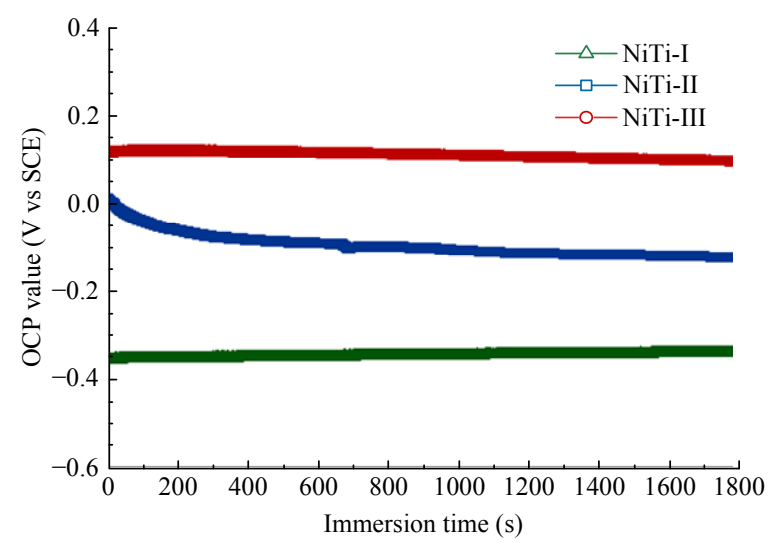

(a)

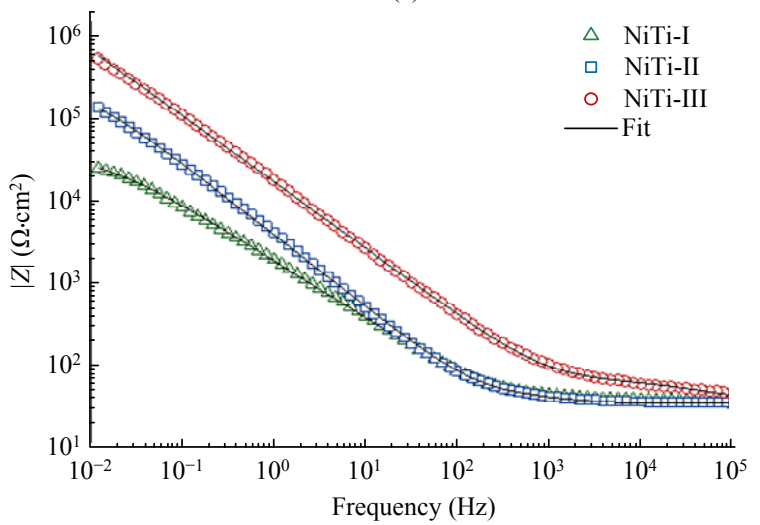

(c)

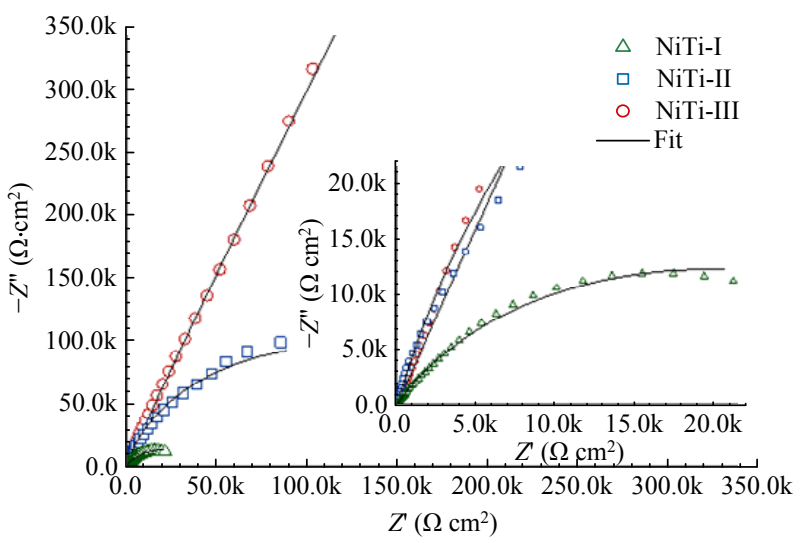

(b)

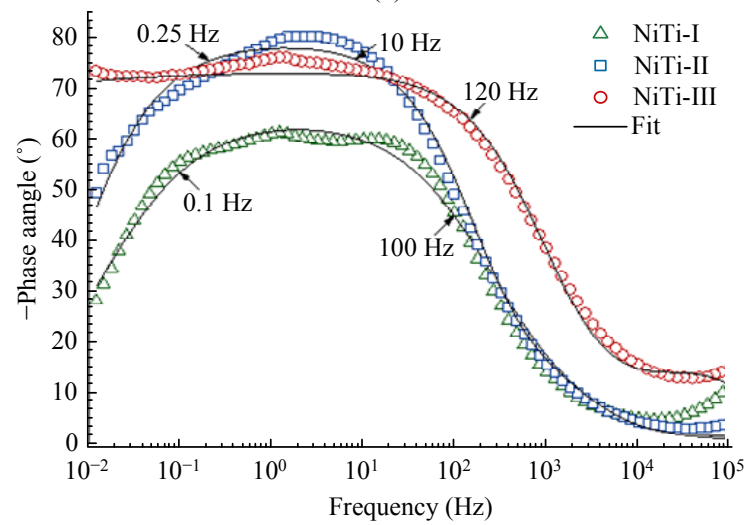

(d)

Fig. 9 EIS results of NiTi-I, NiTi-II and NiTi-III samples in SBF. (a) OCP curves; (b) Nyquist plots and fitted curves; (c) bode plots of impedance modulus versus frequency and fitted curves; (d) bode plots of phase angle versus frequency and fitted curves.

Obviously, the OCP curves stabilized towards a stationary value after a period of immersion. The OCP varied with time, which might be attributed to the changes of the surface nature of working electrodes (oxidation or the formation of passivation layer). The OCP can be employed as an assessment for corrosion behavior.

Fig. $9 \mathrm{~b}$ demonstrated the corresponding Nyquist plots of three kinds of samples, and the illustration figure was the magnified view in the high frequency range. As is known to all, the diameter of the Nyquist loop is a significant parameter to estimate the polarization resistance of the working electrode during electrochemical corrosion process. A bigger diameter of Nyquist loop refers to a higher corrosion resistance. Obviously, it could be found that NiTi-III possessed the widest Nyquist loop, followed by NiTi-II, and NiTi-I presented the smallest Nyquist loop, indicating that the anti-corrosion performance extremely enhanced due to the laser irradiation and PDMS modification. Compared with NiTi-II, NiTi-III possessed a wide Nyquist loop due to the addi- tional presence of super-hydrophobic coating. In addition, the Bode-impedance modulus $|Z|$ versus frequency diagrams of the investigated samples were exhibited in Fig. 9c. Noticeably, NiTi-I had the lowest impedance modulus $|Z|$ at the low frequency of $0.01 \mathrm{~Hz}$, while NiTi-III possessed the biggest impedance modulus $|Z|$. Previous literature had verified that the larger value of impedance modulus $|Z|$ at the low frequency in Bode diagrams implies the better corrosion resistance ${ }^{[42,43]}$. Hence, both the Nyquist plots and Bode diagrams confirmed the excellent corrosion resistance of NiTi-III.

According to the phase angle versus frequency curves shown in Fig. 9d, NiTi-I included one time constant at high frequency range owing to the formation of corrosion layer. In this case, the equivalent circuit in Fig. 10a was adopted and $C P E_{\mathrm{dl}}$ was used to represent the electric double-layer capacitance of interface between the NiTi SMA substrates and electrolyte solution. As for NiTi-II and NiTi-III, two time constants were contained based on the EIS results shown in Fig. 9d: One 
at high frequency and another at low frequency. As shown in Fig. 10b, the equivalent circuit was employed to fit the EIS results of NiTi-II and NiTi-III. The $C P E_{\mathrm{f}}$ was performed as the double electric layer capacitance in the high frequency capacitive loop due to the obstruction of coatings. In the corrosion process, the second time constant related to $C P E_{\mathrm{dl}}$ was generated at the low frequency stage on account of the constant infiltration of electrolyte solution into the coatings. In these fitted equivalent circuits, both $C P E_{\mathrm{dl}}$ and $C P E_{\mathrm{f}}$ are the Constant Phase Element (CPE) replacing pure capacitor, because there are no pure capacitors during the actual electrochemical corrosion process. The following equation was used to calculate the impedance of CPE:

$$
\begin{array}{r}
Z_{\mathrm{CPE}}=\left[Y_{0} \cdot(\mathrm{j} \omega)^{n}\right]^{-1}=Z_{\mathrm{CPE}}^{\prime}+\mathrm{j} Z_{\mathrm{CPE}}^{\prime \prime}, \\
Z_{\mathrm{CPE}}^{\prime}=\left[Y_{0} \cdot \omega^{n}\right]^{-1} \cos \left(\frac{n \pi}{2}\right), \\
Z_{\mathrm{CPE}}^{\prime \prime}=\left[Y_{0} \cdot \omega^{n}\right]^{-1} \sin \left(\frac{n \pi}{2}\right),
\end{array}
$$

where $Z_{\mathrm{CPE}}$ is the impedance of $\mathrm{CPE}, \mathrm{j}=\sqrt{-1}$, implicating that $Z_{\mathrm{CPE}}$ is an imaginary number, $Z_{\mathrm{CPE}}^{\prime}$ and $Z_{\mathrm{CPE}}^{\prime \prime}$ are the real and imaginary parts of the impedance, respectively, $Y_{0}$ is the proportionality factor, $\omega$ denotes the angular frequency, and $n$ is a dimensionless exponent within the range of 0 to $1^{[42]}$.

The equivalent circuit model of NiTi-I samples was shown in Fig. 10a. In this equivalent circuit, $R_{\mathrm{S}}$ describes the electrolyte $(\mathrm{SBF})$ resistance between the reference electrode and working electrode, $C P E_{\mathrm{dl}}$ and $R_{\mathrm{ct}}$ represent the constant phase element and the charge transfer resistance between NiTi SMA substrate and electrolyte solution. As far as NiTi-II and NiTi-III, the better fitting results were obtained via the equivalent circuit displayed in Fig. 10b, where the electrolytic resistance is described by $R_{\mathrm{s}}$, the resistance between the passive film or the PDMS coating and corrosion solution is represented by
$R_{\mathrm{f}}, C P E_{\mathrm{f}}$ means the constant phase element and the corresponding value reflects the number of corrosion ions in contact with the film or coating ${ }^{[43]}$. $C P E_{\mathrm{dl}}$ and $R_{\mathrm{ct}}$ components represent the impedance of interface reaction between the NiTi substrate and the surface coating. The corresponding simulation parameters of circuit elements were summarized in Table 4 . It has been reported that the values of $C P E_{\mathrm{dl}}$ and $R_{\mathrm{ct}}$ are closely related to corrosion resistance. Generally, the increase of charge transfer resistance reveals the better corrosion resistance of as-prepared samples. As shown in Table 4, the $R_{\mathrm{ct}}$ value increased from $3.86 \times 10^{4} \Omega \cdot \mathrm{cm}^{2}$ (NiTi-I samples) to $2.35 \times 10^{5} \Omega \cdot \mathrm{cm}^{2}$ (NiTi-II samples) after laser irradiation, demonstrating that the anti-corrosive property of NiTi SMA surfaces were improved after the ablation process. NiTi-III had the largest $R_{\mathrm{ct}}$ of $3.83 \times 10^{7} \Omega \cdot \mathrm{cm}^{2}$, revealing that the ions such as $\mathrm{Cl}^{-}, \mathrm{HCO}^{3-}$ and $\mathrm{HPO}^{2-}$ in SBF serving as corrosive medium had enormous drag to permeate the super-hydrophobic coatings. Compared with NiTi-I, the $C P E_{\mathrm{dl}}$ value of NiTi-II decreased by about four orders of magnitude with the value of $3.87 \times$ $10^{-5} \Omega^{-1} \cdot \mathrm{s}^{n} \cdot \mathrm{cm}^{-2}$. The corresponding value of NiTi-III had a marked down of five orders of magnitude. Obviously, this result confirmed the fewer corrosive ions in SBF touching with inner NiTi SMA during electrochemical measurement compared with NiTi-II. Besides, the release concentration of $\mathrm{Ni}$ ions from aforesaid samples

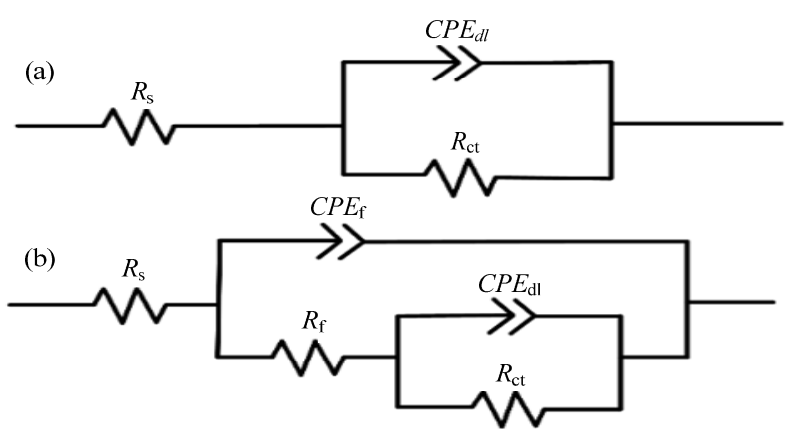

Fig. 10 Equivalent circuit models matched to the EIS plots of (a)

\begin{tabular}{|c|c|c|c|c|c|c|c|}
\hline \multirow{2}{*}{ Sample } & \multirow{2}{*}{$\begin{array}{c}R_{\mathrm{s}} \\
\Omega \cdot \mathrm{cm}^{2}\end{array}$} & \multirow{2}{*}{$\begin{array}{c}R_{\mathrm{ct}} \\
\Omega \cdot \mathrm{cm}^{2}\end{array}$} & \multicolumn{2}{|c|}{$C P E_{\mathrm{dl}}$} & \multirow{2}{*}{$\begin{array}{c}R_{\mathrm{f}} \\
\Omega \cdot \mathrm{cm}^{2}\end{array}$} & \multicolumn{2}{|c|}{$C P E_{\mathrm{f}}$} \\
\hline & & & $Q_{\mathrm{dl}}$ & $n$ & & $Q_{\mathrm{f}}$ & $n$ \\
\hline NiTi-I & 33.1 & $3.86 \times 10^{4}$ & 0.149 & 0.72 & - & - & - \\
\hline NiTi-II & 33.3 & $2.35 \times 10^{5}$ & $3.87 \times 10^{-5}$ & 0.84 & $2.16 \times 10^{3}$ & $1.56 \times 10^{-5}$ & 0.94 \\
\hline NiTi-III & 34.3 & $3.83 \times 10^{7}$ & $9.54 \times 10^{-6}$ & 0.84 & $3.36 \times 10^{4}$ & $4.43 \times 10^{-6}$ & 0.73 \\
\hline
\end{tabular}
NiTi-I, (b) NiTi-II and NiTi-III samples.

Table 4 The parameters abstracted from the Nyquist plots by fitting the equivalent circuit model 


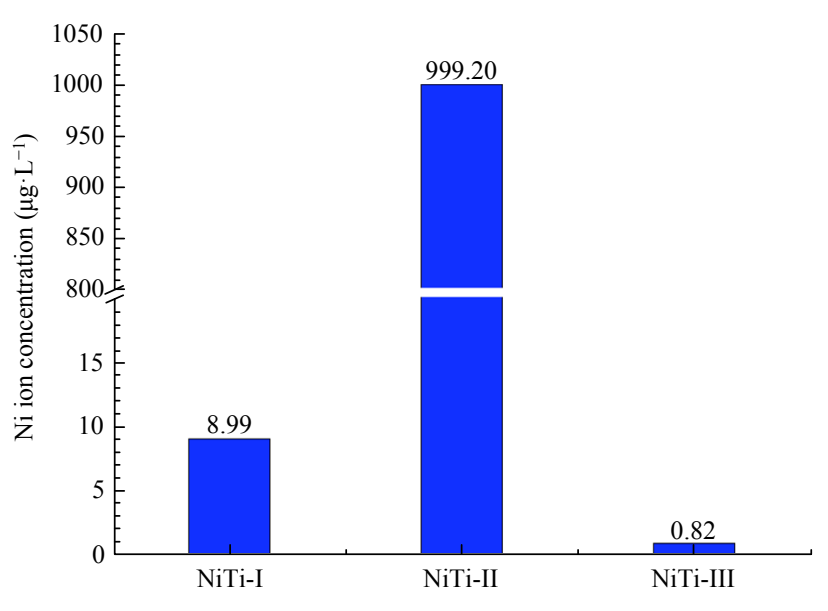

Fig. 11 The concentration of Ni ions released from NiTi-I, NiTi-II and NiTi-III samples after immersion in SBF for $4 \mathrm{~h}$.

was obtained by ICP-MS after electrochemical corrosion process. The corresponding results and statements are shown in Fig. S1.

The above results sufficiently demonstrated that the laser irradiation technique could effectively prevent corrosive ions penetrating into the substrates, enhancing the anti-corrosion of NiTi SMA. The main reason is that titanium oxide layers were produced on NiTi SMA surfaces during the laser irradiation process. The titanium oxide layer was regarded as the passive film to restrain the transport of corrosion ions and the dissolution of NiTi SMA ${ }^{[44]}$. Among three kinds of investigated samples, NiTi-III samples showed the most outstanding corrosion resistance. Because this kind surface possessed two-tier protective layers: fresh laser-induced titanium oxide layer and PDMS modified super-hydrophobic layer. Apart from the protection of titanium oxide layer, the PDMS modified layer could extremely decrease the contact area between the sample surfaces and the corrosive medium, due to its splendid low-affinity to water.

\subsection{Ni ions release}

As shown in Fig. 11, the concentration of $\mathrm{Ni}$ ions released from NiTi-I, NiTi-II and NiTi-III samples were compared using ICP-MS after immersion in SBF for $4 \mathrm{~h}$ at room temperature and ambient air. It is apparent that the amount of Ni ions released from NiTi-III with PDMS layer was about 10 times and 1220 times smaller than those from NiTi-I and NiTi-II. The super-hydrophobic coating was effective greatly in mitigating $\mathrm{Ni}$ ions

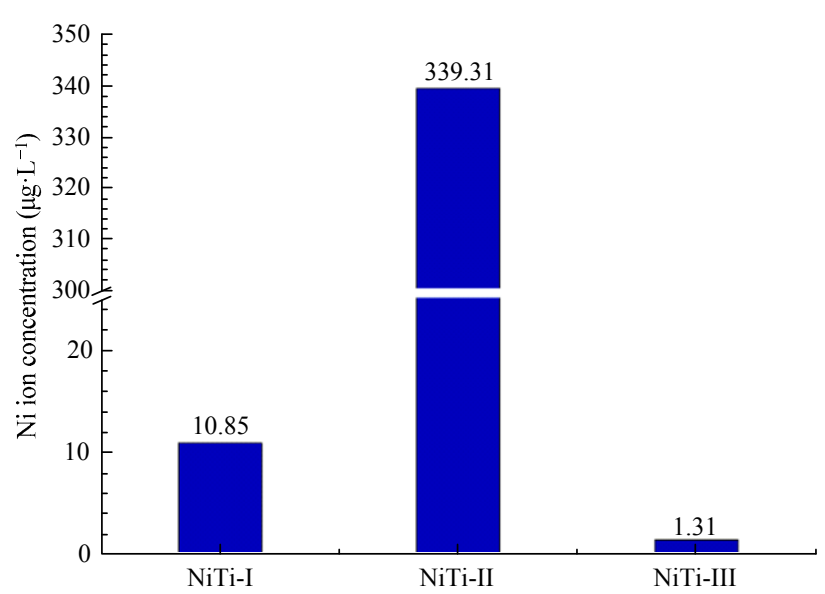

Fig. 12 The concentration of Ni ions released from NiTi-I, NiTi-II and NiTi-III samples after immersion in PBS for $2 \mathrm{~h}$.

release out of NiTi substrates. It can be deduced that the main reason for reduction of $\mathrm{Ni}$ ions release might be related with the decrease of the contact area between samples and corrosion ions. As far as NiTi-II, there was the highest amount of $\mathrm{Ni}$ ions release due to the largest solid-liquid contact area and the surface imperfection after laser ablation. Hence, the hybrid of ns-laser irradiation and PDMS modification is a feasible method to reduce ions release from NiTi SMA substrates.

\subsection{Analysis of immersion extracts}

The immersion extracts were obtained after the investigated samples were immersed in PBS for $2 \mathrm{~h}$ in the aseptic conditions. Fig. 12 exhibited the nickel ion concentration results of the three kinds of immersion extracts. Obviously, NiTi-III released the fewest amount of Ni ions, followed by NiTi-I, and NiTi-II exhibited the maximum, indicating that the fabricated super-hydrophobic surfaces effectively reduced the release of $\mathrm{Ni}$ ions. As a kind of metal ion with potentially toxicity, $\mathrm{Ni}$ ions can induce the malformation of cells and even apoptosis ${ }^{[4]}$. Previous researches have demonstrated that the concentration of $\mathrm{Ni}$ ions in immersion extracts definitively impacts the cell viability: The lower nickel ion concentration results in the higher cell survival rate. On the contrary, the higher concentration leads to the lower cell survival rate ${ }^{[45]}$. Based on the theoretical support of previous literatures, it can be predicted that the fabricated super-hydrophobic surfaces could effectively mitigate $\mathrm{Ni}$-induced cytotoxicity and improve the cytocompatibility of NiTi SMA with the 
good cell viability, which was attributed to the super-hydrophobic coatings reducing the release amount of Ni ions.

\section{Conclusion}

A new and safe technique combing the ns-laser processing with PDMS modification was proposed to fabricate the super-hydrophobic surfaces on the NiTi SMA samples.

(1) The surfaces of laser-induced samples showed super-hydrophilicity, the wettability of which became super-hydrophobic after chemical modification with PDMS modifier. The super-hydrophobic surfaces exhibited high WCA of $155.4^{\circ} \pm 0.9^{\circ}$ and small WSA of $4.4^{\circ}$ $\pm 1.1^{\circ}$.

(2) The results of SEM and XPS revealed the formation mechanism of super-hydrophobic surfaces, owing to the combined action of the micro-nano texture and PDMS with low free energy.

(3) The electrochemical corrosion test and immersion test showed that the PDMS super-hydrophobic coatings exhibited superior corrosion resistance and it could serve as a protection layer for effectively inhibiting $\mathrm{Ni}$ ions release.

(4) The analysis results of cell viability displayed that the PDMS super-hydrophobic coatings could improve the biocompatibility of NiTi SMA and promote the biomedical application of the coatings.

It is believed that our work would serve as significant reference on anti-corrosion methods selection in medical. The research including mechanical stability, cellular biocompatibility and blood compatibility will be studied in our future work. What's more, the super-hydrophobic surface will be fabricated on a medical device surface-scalpel via this safe processing method in this work. The potential application value in medical would be further delved.

\section{Acknowledgment}

The authors thank very much for receiving funding support from National Key R\&D Program of China (No. 2017YFB1104700) and the National Natural Science Foundations of China (Nos. 51675371, 51675376 and 51675367).

* All supplementary materials are available at https://doi.org/10.1007/s42235-021-0011-5.

Open Access This article is licensed under a Creative Commons Attribution 4.0 International License, which permits use, sharing, adaptation, distribution and reproduction in any medium or format, as long as you give appropriate credit to the original author(s) and the source, provide a link to the Creative Commons licence, and indicate if changes were made.

The images or other third party material in this article are included in the article's Creative Commons licence, unless indicated otherwise in a credit line to the material. If material is not included in the article's Creative Commons licence and your intended use is not permitted by statutory regulation or exceeds the permitted use, you will need to obtain permission directly from the copyright holder.

To view a copy of this licence, visit http://creativecommons.org/licenses/by/4.0/.

\section{References}

[1] Khalili V, Naji H. Developing a mechanochemical surface pretreatment to increase the adhesion strength of hydroxyapatite electrophoretic coating on the NiTi alloy as a bone implant. Surface and Coatings Technology, 2020, 397, 125985 .

[2] Li J, Chen D S, Zhang Y Y, Yao Y, Mo Z J, Wang L Z, Fan Y B. Diagonal-symmetrical and mid-line-symmetrical unit cells with same porosity for bone implant: Mechanical properties evaluation. Journal of Bionic Engineering, 2019, 16, 468-479.

[3] Wang M, Yang Z, Yang C J, Zhang D W, Tian Y L, Liu X P. The investigation of mechanical and thermal properties of super-hydrophobic nitinol surfaces fabricated by hybrid methods of laser irradiation and carbon ion implantation. Applied Surface Science, 2020, 527, 146889.

[4] Genchi G, Carocci A, Lauria G, Sinicropi M S, Catalano A. Nickel: Human health and environmental toxicology. International Journal of Environmental Research and Public Health, 2020, 17, 679.

[5] Srimaneepong V, Rokaya D, Thunyakitpisal P, Qin J Q, Saengkiettiyut K. Corrosion resistance of graphene oxide/silver coatings on Ni-Ti alloy and expression of IL-6 and IL-8 in human oral fibro-blasts. Scientific Reports, 2020, 10, 3247.

[6] Weng Z M, Bai L, Liu Y L, Zhao Y, Sun Y H, Zhang X Y, Huang X B, Huang D, Yao X H, Huang R Q. Osteogenic 
activity, antibacterial ability, and $\mathrm{Ni}$ release of Mg-incorporated Ni-Ti-O nanopore coatings on NiTi alloy. Applied Surface Science, 2019, 486, 441-451.

[7] Tohidi P M S, Safavi M S, Etminanfar M, Khalil-Allafi J. Pulsed electrodeposition of compact, corrosion resistant, and bioactive HAp coatings by application of optimized magnetic field. Materials Chemistry and Physics, 2020, 254, 123511.

[8] Kurtoglu S F, Yagci M B, Uzun A, Unal U, Canadinc D. Enhancing biocompatibility of NiTi shape memory alloys by simple $\mathrm{NH}_{3}$ treatments. Applied Surface Science, 2020, 525, 146547.

[9] Peng J Y, Zhao X J, Wang W F, Gong X. Durable self-cleaning surfaces with superhydrophobic and highly oleophobic properties. Langmuir, 2019, 35, 8404-8412.

[10] Liu X, Zhang D K, Guo Z G. A facile modifier-free approach to fabricate antistatic superhydrophobic composite coatings with remarkable thermal stability and corrosion resistance. Journal of Bionic Engineering, 2020, 17, 421-435.

[11] Xu S S, Wang Q, Wang N. Eco-friendly fabrication of super-hydrophobic surface with anti-corrosion by transferring dendrite-like structures to aluminum substrate. Colloids and Surfaces A, 2020, 595, 124719.

[12] Yang X N, Tian L M, Wang W, Fan Y, Sun J Y, Zhao J, Ren L Q. Bio-inspired superhydrophobic self-healing surfaces with synergistic anticorrosion performance. Journal of Bionic Engineering, 2020, 17, 1-13.

[13] Xin G Q, Wu C Y, Cao H Y, Liu W N, Li B, Huang Y, Rong Y M, Zhang G J. Superhydrophobic $\mathrm{TC}_{4}$ alloy surface fabricated by laser micro-scanning to reduce adhesion and drag resistance. Surface and Coatings Technology, 2020, 391, 125707.

[14] Liu X, Zhang T C, He H Q, Ouyang L K, Yuan S J. A stearic acid/ $\mathrm{CeO}_{2}$ bilayer coating on $\mathrm{AZ}_{31} \mathrm{~B}$ magnesium alloy with superhydrophobic and self-cleaning properties for corrosion inhibition. Journal of Alloys and Compounds, 2020, 834, 155210 .

[15] Zhang Y J, Sun D, Shen J L, Xin X. Dynamic self-assembly of silver nanoclusters into luminescent nanotubes with controlled surface roughness: Scaffold of superhydrophobic materials. Applied Surface Science, 2020, 514, 145913.

[16] Yang Z, Liu X P, Tian Y L. A contrastive investigation on anticorrosive performance of laser-induced super-hydrophobic and oil-infused slippery coatings. Progress in Organic Coatings, 2020, 138, 105313.

[17] Tas M, Xu F, Ahmed I, Hou X H. One-step fabrication of superhydrophobic $\mathrm{P}(\mathrm{VDF}-\mathrm{co}-\mathrm{HFP})$ nanofiber membranes using electrospinning technique. Journal of Applied Polymer Science, 2019, 137, 48817.

[18] Yang Z, Liu X P, Tian Y L. Novel metal-organic super-hydrophobic surface fabricated by nanosecond laser irradiation in solution. Colloids and Surfaces A, 2020, 587, 124343.

[19] Zhang H, Lu X, Xin Z, Zhang W F, Zhou C L. Preparation of superhydrophobic polybenzoxazine $/ \mathrm{SiO}_{2}$ films with self-cleaning and ice delay properties. Progress in Organic Coatings, 2018, 123, 254-260.

[20] Li D W, Wang H Y, Liu Y, Wei D S, Zhao Z X. Large-scale fabrication of durable and robust super-hydrophobic spray coatings with excellent repairable and anti-corrosion performance. Chemical Engineering Journal, 2019, 367, 169-179.

[21] Rong Y M, Huang Y, Lin C R, Liu Y F, Shi S X, Zhang G J, Wu C Y. Stretchability improvement of flexiable electronics by laser micro-drilling array holes in PDMS film. Optics and Lasers in Engineering, 2020, 134, 106307.

[22] Wang H P, He M J, Liu H, Guan Y C. One-step fabrication of robust superhydrophobic steel surfaces with mechanical durability, thermal stability, and anti-icing function. ACS Applied Materials and Interfaces, 2019, 11, 25586-25594.

[23] Xin G Q, Wu C Y, Cao H Y, Liu W N, Li B, Huang Y, Rong Y M, Zhang G J. Superhydrophobic $\mathrm{TC}_{4}$ alloy surface fabricated by laser micro-scanning to reduce adhesion and drag resistance. Surface and Coatings Technology, 2020, 391, 125707.

[24] Li J R, Xu J K, Lian Z X, Yu Z J, Yu H D. Fabrication of antireflection surfaces with superhydrophobic property for titanium alloy by nanosecond laser irradiation. Optics and Laser Technology, 2020, 126, 106129.

[25] Yang Z, Tian Y L, Zhao Y C, Yang C J. Study on the fabrication of super-hydrophobic surface on Inconel alloy via nanosecond laser ablation. Materials, 2019, 12, 278.

[26] Meng W, Zhang D W, Yang Z, Yang C J, Tian Y L, Liu X P. A contrastive investigation on the anticorrosive performance of stearic acid and fluoroalkylsilane-modified superhydrophobic surface in salt, alkali, and acid solution. Langmuir, 2020, 36, 10279-10292.

[27] Long J Y, Zhong M L, Zhang H J, Fan P X. Superhydrophilicity to superhydrophobicity transition of picosecond laser microstructured aluminum in ambient air. Journal of Colloid and Interface Science, 2019, 441, 1-9.

[28] Gao X Y, Guo Z G. Mechanical stability, corrosion resistance of superhydrophobic steel and repairable durability of its slippery surface. Journal of Colloid and Interface Science, 
2018, 512, 239-248.

[29] Jian R, Pitchumani R. Facile fabrication of durable copper-based superhydrophobic surfaces via electrodeposition. Langmuir, 2018, 34, 3159-3169.

[30] He S J, Wang Z, Hu J, Zhu J B, Wei L P, Chen Z. Formation of superhydrophobic micro-nanostructured iron oxide for corrosion protection of N80 steel. Materials and Design, 2018, 160, 84-94.

[31] Lu Y, Guan Y C, Li Y, Yang L J, Wang M L, Wang Y. Nanosecond laser fabrication of superhydrophobic surface on $316 \mathrm{~L}$ stainless steel and corrosion protection application. Colloids and Surfaces A, 2020, 604, 125259.

[32] Li D W, Wang H Y, Luo D, Liu Y, Han Z W, Ren L Q. Corrosion resistance controllable of biomimetic super-hydrophobic microstructured magnesium alloy by controlled adhesion. Surface and Coatings Technology, 2018, 347, 173-180.

[33] Lin C, Huang Y F, Li X, Sun X, Zhang W Y, Huan, J Y, Ying X G, Liu M H. Fabrication of superhydrophobic surfaces inspired by "stomata effect" of plant leaves via swelling-vesiculating-cracking method. Chemical Engineering Journal, 2020, 400, 125935.

[34] Yang Z, Tian Y L, Yang C J, Wang F J, Liu X P. Modification of wetting property of Inconel 718 surface by nanosecond laser texturing. Applied Surface Science, 2017, 414, 313-324.

[35] Yang Z, Liu X P, Tian Y L. Hybrid laser ablation and chemical modification for fast fabrication of bio-inspired super-hydrophobic surface with excellent self-cleaning, stability and corrosion resistance. Journal of Bionic Engineering, 2019, 16, 13-26.

[36] Bao W N, Liang X D, Liu Y Y, Gao Y F, Wang J F. Effects of AC and DC corona on the surface properties of silicone rubber: Characterization by contact angle measurements and XPS high resolution scan. IEEE Transactions on Dielectrics and Electrical Insulation, 2017, 24, 2911-2919.
[37] Peng C, Zhang C X, Lv M, Wu J Q. Preparation of silica encapsulated carbon black with high thermal stability. $\mathrm{Ce}$ ramics International, 2013, 39, 7247-7253.

[38] Katsiev K, Harrison G, Alghamdi H, Alsalik Y, Wilson A, Thornton G, Idriss H. Mechanism of ethanol photooxidation on single-crystal anatase $\mathrm{TiO}_{2}(101)$. Journal of Physical Chemistry C, 2017, 121, 2940-2950.

[39] Hass K C, Schneider W F, Curioni A, Andreoni W. The chemistry of water on alumina surfaces: Reaction dynamics from first principles. Science, 1998, 282, 265-268.

[40] Argyris D, Ashby P D, Striolo A. Structure and orientation of interfacial water determine atomic force microscopy results: Insights from molecular dynamics simulations. ACS Nano, 2011, 5, 2215-2223.

[41] Chembath M, Balaraju J N, Sujata M. Surface characteristics, corrosion and bioactivity of chemically treated biomedical grade NiTi alloy. Materials Science and Engineering $C$, 2015, 56,417-425.

[42] Liu Y, Li S Y, Zhang J J, Liu J A, Han Z W, Ren L Q. Corrosion inhibition of biomimetic super-hydrophobic electrodeposition coatings on copper substrate. Corrosion Science, 2015, 94, 190-196.

[43] Yang C J, Wang M, Yang Z, Zhang D W, Tian Y L, Jing X B, Liu X P. Investigation of effects of acid, alkali, and salt solutions on fluorinated superhydrophobic surfaces. Langmuir, 2019, 35, 17027-17036

[44] Cui Z D, Man H C, Yang X J. The corrosion and nickel release behavior of laser surface-melted NiTi shape memory alloy in Hanks, solution. Surface and Coatings Technology, 2005, 192, 347-353.

[45] Meng L, Wu Y A, Pan K, Zhu Y, Li X J, Wei W, Liu X. Polymeric nanoparticles-based multi-functional coatings on NiTi alloy with nickel ion release control, cytocompatibility, and antibacterial performance. New Journal of Chemistry, 2019, 43, 1551-1561. 